\title{
Canonical Connection on a Class of Rieman- nian Almost Product Manifolds
}

\author{
Dobrinka Gribacheva and Dimitar Mekerov
}

\begin{abstract}
The canonical connection on a Riemannian almost product manifold is an analogue to the Hermitian connection on an almost Hermitian manifold. In this paper we consider the canonical connection on a class of Riemannian almost product manifolds with non-integrable almost product structure.
\end{abstract}

Mathematics Subject Classification (2010). 53C15, 53C25, 53C05, 53C07, $22 \mathrm{E} 60$.

Keywords. Riemannian almost product manifold, nonintegrable structure, canonical connection, parallel torsion, Lie group, Killing metric.

\section{Introduction}

On an Hermitian manifold $(M, J, g)$ there exists an unique linear connection $D$ with a torsion $T$ such that $D J=D g=0$ and $T(x, J y)=T(J x, y)$ for all vector fields $x, y$ on $M$. This is the Hermitian connection of the manifold ( 1 , 2, 3]). The group of the conformal transformations of the metric $g$ generates the conformal group of the transformations of $D$.

Analogously to the Hermitian connection on an almost Hermitian manifold, $\mathrm{V}$. Mihova in [4] define on a Riemannian almost product manifold $(M, P, g)$ a natural connection $\nabla^{\prime}$ (i.e. $\nabla^{\prime} P=\nabla^{\prime} g=0$ ) with torsion $T$ satisfying $T(x, y, z)+T(y, z, x)+T(P x, y, P z)+T(y, P z, P x)=0$. This connection is called canonical and it is proved that it is unique on $(M, P, g)$.

The systematic development of the theory of Riemannian almost product manifolds was started by K. Yano [5]. In 6] A. M. Naveira gives a classification of these manifolds with respect to the covariant differentiation of the

This paper is partially supported by the project NI11-FMI-004 of the Scientific Research Fund at University of Plovdiv, Bulgaria. 
almost product structure. Having in mind the results in [6], M. Staikova and K. Gribachev give in 7 a classification of the Riemannian almost product manifolds $(M, P, g)$ with $\operatorname{tr} P=0$. The intersection of the basic classes in this classification is the class $\mathcal{W}_{0}$ of the Riemannian $P$-manifolds determined by $\nabla P=0$, where $\nabla$ is the Levi-Civita connection.

In the present work we consider the canonical connection on the manifolds of the class $\mathcal{W}_{3}$ from the Staikova-Gribachev classification which we called Riemannian almost product $\mathcal{W}_{3}$-manifolds. The class $\mathcal{W}_{3}$ is the only basic class where any manifold $(M, P, g) \notin \mathcal{W}_{0}$ has a non-integrable almost product structure $P$. This class is an object of interest in 8 .

In Section 2 we give some necessary facts about the class $\mathcal{W}_{3}$. We introduce the notion of a Riemannian $P$-tensor $L$ which is curvature-like and has the property $L(x, y, P z, P w)=L(x, y, z, w)$. This tensor is an analogue of the Kähler tensor in Hermitian geometry.

In Section 3 we recall facts about the natural connections (i.e. the connections preserving $P$ and $g$ ) with torsion on Riemannian almost product manifolds $(M, P, g)$. We find conditions for the torsion of such a connection when $(M, P, g)$ is a Riemannian almost product $\mathcal{W}_{3}$-manifold.

In Section 4 we consider the canonical connection $\nabla^{\prime}$ on a Riemannian almost product $\mathcal{W}_{3}$-manifold $(M, P, g)$. We find properties of the torsion of $\nabla^{\prime}$ as well as the exact expression of $\nabla^{\prime}$. Let us point out the result in Theorem 4.3. where the tensor norm $\|\nabla P\|^{2}$ is given in terms of the scalar curvatures of $\nabla$ and $\nabla^{\prime}$. Due to the definitness of the metric $g$, the important Corollary 4.4 is obtained.

In Section 5 we establish properties of a Riemannian almost product $\mathcal{W}_{3}$ manifold for which the curvature tensor of the canonical connection is a Riemannian $P$-tensor.

In Section 6 we study the case of canonical connection $\nabla^{\prime}$ with parallel torsion $T$ on a Riemannian almost product $\mathcal{W}_{3}$-manifold. The relation between the curvature tensors of $\nabla$ and $\nabla^{\prime}$ is found. An important result is given in Theorem 6.4, where we prove that a necessary condition for the curvature tensor of $\nabla^{\prime}$ to be a Riemannian $P$-tensor is the parallelism of the torsion $T$ with respect to $\nabla^{\prime}$.

In Section 7 we consider a 4 -dimensional Riemannian almost product $\mathcal{W}_{3}$ manifold $(G, P, g)$, where $G$ is a Lie group. The properties of Riemannian almost product manifolds $(G, P, g)$ are expressed in terms of the commutators in the corresponding Lie algebra. At first, we find some geometrical characteristics of the manifold $(G, P, g)$. After that, we interpret theoretical results obtained in this paper in accordance with the canonical connection on $(G, P, g)$. 


\section{Riemannian almost product $\mathcal{W}_{3}$-manifolds}

Let $(M, P, g)$ be a Riemannian almost product manifold, i.e. a differentiable manifold $M$ with a tensor field $P$ of type $(1,1)$ and a Riemannian metric $g$ such that

$$
P^{2} x=x, \quad g(P x, P y)=g(x, y)
$$

for arbitrary $x, y$ of the algebra $\mathfrak{X}(M)$ of the smooth vector fields on $M$. Obviously $g(P x, y)=g(x, P y)$.

Further $x, y, z, w$ will stand for arbitrary elements of $\mathfrak{X}(M)$ or vectors in the tangent space $T_{p} M$ at $p \in M$.

In this work we consider Riemannian almost product manifolds with $\operatorname{tr} P=0$. In this case $(M, P, g)$ is an even-dimensional manifold. If $\operatorname{dim} M=2 n$ then the associated metric $\tilde{g}$ of $g$, determined by $\tilde{g}(x, y)=g(x, P y)$, is an indefinite metric of signature $(n, n)$. Since $\tilde{g}(P x, P y)=\tilde{g}(x, y)$, the manifold $(M, P, \tilde{g})$ is a pseudo-Riemannian almost product manifold.

The classification in [7] of Riemannian almost product manifolds with $\operatorname{tr} P=0$ is made with respect to the tensor $F$ of type $(0,3)$, defined by

$$
F(x, y, z)=g\left(\left(\nabla_{x} P\right) y, z\right),
$$

where $\nabla$ is the Levi-Civita connection of $g$. The tensor $F$ has the following properties:

$$
F(x, y, z)=F(x, z, y)=-F(x, P y, P z), F(x, y, P z)=-F(x, P y, z) .
$$

The basic classes of the classification in 7] are $\mathcal{W}_{1}, \mathcal{W}_{2}$ and $\mathcal{W}_{3}$. Their intersection is the class $\mathcal{W}_{0}$ of the Riemannian $P$-manifolds, determined by the condition $F=0$ or equivalently $\nabla P=0$. In the classification there are include the classes $\mathcal{W}_{1} \oplus \mathcal{W}_{2}, \mathcal{W}_{1} \oplus \mathcal{W}_{3}, \mathcal{W}_{2} \oplus \mathcal{W}_{3}$ and the class $\mathcal{W}_{1} \oplus \mathcal{W}_{2} \oplus \mathcal{W}_{3}$ of all Riemannian almost product manifolds.

In the present work we consider the manifolds from the class $\mathcal{W}_{3}$. This class is determined by the condition

$$
\underset{x, y, z}{\mathfrak{S}} F(x, y, z)=0,
$$

where $\mathfrak{S}_{x, y, z}$ is the cyclic sum by $x, y, z$. This is the only class of the basic classes $\mathcal{W}_{1}, \mathcal{W}_{2}$ and $\mathcal{W}_{3}$, where each manifold (which is not a Riemannian $P$ manifold) has a nonintegrable almost product structure $P$, i.e. the Nijenhuis tensor $N$, determined by

$$
N(x, y)=\left(\nabla_{x} P\right) P y-\left(\nabla_{y} P\right) P x+\left(\nabla_{P x} P\right) y-\left(\nabla_{P y} P\right) x,
$$

is non-zero.

In [7] it is introduced an associated tensor $N^{*}$ by

$$
N^{*}(x, y)=\left(\nabla_{x} P\right) P y+\left(\nabla_{P x} P\right) y+\left(\nabla_{y} P\right) P x+\left(\nabla_{P y} P\right) x .
$$

It is proved that the condition (2.4) is equivalent to $N^{*}(x, y)=0$. 
Further, manifolds of the class $\mathcal{W}_{3}$ we call Riemannian almost product $\mathcal{W}_{3}$ manifolds.

As it is known the curvature tensor $R$ of a Riemannian manifold with metric $g$ is determined by $R(x, y) z=\nabla_{x} \nabla_{y} z-\nabla_{y} \nabla_{x} z-\nabla_{[x, y]} z$ and the corresponding $(0,4)$-tensor is defined as follows $R(x, y, z, w)=g(R(x, y) z, w)$.

Let $(M, P, g)$ be a Riemannian almost product manifold and $\left\{e_{i}\right\}$ be a basis of $T_{p} M$. Let the components of the inverse matrix of $g$ with respect to $\left\{e_{i}\right\}$ be $g^{i j}$. Then the quantities $\rho$ and $\tau$, determined by $\rho(y, z)=g^{i j} R\left(e_{i}, y, z, e_{j}\right)$ and $\tau=g^{i j} \rho\left(e_{i}, e_{j}\right)$, are the Ricci tensor and the scalar curvature for $\nabla$, respectively.

The square norm of $\nabla P$ is defined by

$$
\|\nabla P\|^{2}=g^{i j} g^{k s} g\left(\left(\nabla_{e_{i}} P\right) e_{k},\left(\nabla_{e_{j}} P\right) e_{s}\right) .
$$

Obviously $\|\nabla P\|^{2}=0$ if and only if $(M, P, g)$ is a Riemannian $P$-manifold.

A tensor $L$ of type $(0,4)$ with properties

$$
\begin{gathered}
L(x, y, z, w)=-L(y, x, z, w)=-L(x, y, w, z), \\
\underset{x, y, z}{\mathfrak{S}} L(x, y, z, w)=0 \quad \text { (the first Bianchi identity), } \\
L(x, y, P z, P w)=L(x, y, z, w),
\end{gathered}
$$

is called a Riemannian P-tensor.

\section{Natural connection on Riemannian almost product manifolds}

The linear connections in our investigations have a torsion.

Let $\nabla^{\prime}$ be a linear connection determined by $\nabla_{x}^{\prime} y=\nabla_{x} y+Q(x, y)$, where $Q$ is a $(1,2)$-tensor. The torsion $(1,2)$-tensor $T$ is determined by $T(x, y)=$ $\nabla_{x}^{\prime} y-\nabla_{y}^{\prime} x-[x, y]$. The corresponding $(0,3)$-tensors are defined by

$$
Q(x, y, z)=g(Q(x, y), z), \quad T(x, y, z)=g(T(x, y), z) .
$$

The symmetry of the Levi-Civita connection implies

$$
\begin{gathered}
T(x, y)=Q(x, y)-Q(y, x), \\
T(x, y)=-T(y, x) .
\end{gathered}
$$

A partial decomposition of the space $\mathcal{T}$ of the torsion tensors $T$ of type $(0,3)$ is valid on a Riemannian almost product manifold $(M, P, g): \mathcal{T}=\mathcal{T}_{1} \oplus \mathcal{T}_{2} \oplus$ 
$\mathcal{T}_{3} \oplus \mathcal{T}_{4}$, where $\mathcal{T}_{i}(i=1,2,3,4)$ are invariant orthogonal subspaces 4 . For the projection operators $p_{i}$ of $\mathcal{T}$ in $\mathcal{T}_{i}$ is established:

$$
\begin{aligned}
p_{1}(x, y, z)=\frac{1}{8}\{ & 2 T(x, y, z)-T(y, z, x)-T(z, x, y)-T(P z, x, P y) \\
& +T(P y, z, P x)+T(z, P x, P y)-2 T(P x, P y, z) \\
& +T(P y, P z, x)+T(P z, P x, y)-T(y, P z, P x)\} \\
p_{2}(x, y, z)=\frac{1}{8}\{2 T(x, y, z)+T(y, z, x)+T(z, x, y)+T(P z, x, P y) & -T(P y, z, P x)-T(z, P x, P y)-2 T(P x, P y, z) \\
& -T(P y, P z, x)-T(P z, P x, y)+T(y, P z, P x)\}, \\
p_{3}(x, y, z)=\frac{1}{4}\{T(x, y, z)+T(P x, P y, z)-T(P x, y, P z) & -T(x, P y, P z)\}, \\
p_{4}(x, y, z)=\frac{1}{4}\{ & T(x, y, z)+T(P x, P y, z)+T(P x, y, P z) \\
+ & T(x, P y, P z)\} .
\end{aligned}
$$

Definition 3.1 (4]). A linear connection $\nabla^{\prime}$ on a Riemannian almost product manifold $(M, P, g)$ is called a natural connection if $\nabla^{\prime} P=\nabla^{\prime} g=0$ (or equivalently $\left.\nabla^{\prime} g=\nabla^{\prime} \tilde{g}=0\right)$.

If $\nabla^{\prime}$ is a linear connection with a $(0,3)$-tensor $Q$ on a Riemannian almost product manifold, then it is a natural connection if and only if the following conditions are valid [4:

$$
\begin{gathered}
F(x, y, z)=Q(x, y, P z)-Q(x, P y, z), \\
Q(x, y, z)=-Q(x, z, y) .
\end{gathered}
$$

Let $\Phi$ be the (0,3)-tensor determined by

$$
\Phi(x, y, z)=g\left(\widetilde{\nabla}_{x} y-\nabla_{x} y, z\right),
$$

where $\widetilde{\nabla}$ is the Levi-Civita connection of the associated metric $\tilde{g}$.

Theorem 3.2 ([4]). A linear connection with the torsion $T$ on a Riemannian almost product manifold $(M, P, g)$ is natural if and only if

$$
\begin{aligned}
4 p_{1}(x, y, z)= & -\Phi(x, y, z)+\Phi(y, z, x)-\Phi(x, P y, P z) \\
& -\Phi(y, P z, P x)+2 \Phi(z, P x, P y),
\end{aligned}
$$




$$
4 p_{3}(x, y, z)=-g(N(x, y), z)=-2\{\Phi(z, P x, P y)+\Phi(z, x, y)\} .
$$

Proposition 3.3. For the torsion $T$ of a natural connection on a Riemannian almost product $\mathcal{W}_{3}$-manifold $(M, P, g) \notin \mathcal{W}_{0}$, the following properties are valid

$$
p_{1}=0, \quad p_{3} \neq 0 .
$$

Proof. In [7] it is proved that the both basic tensors $F$ and $\Phi$ on a Riemannian almost product manifold $(M, P, g)$ are related as follows:

$$
\Phi(x, y, z)=\frac{1}{2}\{-F(P z, x, y)+F(x, y, P z)+F(y, P z, x)\} .
$$

If $(M, P, g)$ is a Riemannian almost product $\mathcal{W}_{3}$-manifold then (2.3), (2.4) and (3.7) imply

$$
\Phi(x, y, z)=-F(P z, x, y) .
$$

From equalities (3.8), (3.5), (2.3), (2.4) we get $p_{1}=0$. Since $N \neq 0$ for a Riemannian almost product $\mathcal{W}_{3}$-manifold $(M, P, g) \notin \mathcal{W}_{0}$, then (3.6) implies $p_{3} \neq 0$.

\section{Canonical connection on Riemannian almost product $\mathcal{W}_{3}$-manifolds}

Definition 4.1 (4]). A natural connection with torsion $T$ on a Riemannian almost product manifold $(M, P, g)$ is called a canonical connection if

$$
T(x, y, z)+T(y, z, x)+T(P x, y, P z)+T(y, P z, P x)=0 .
$$

In [4] it is shown that (4.1) is equivalent to the condition

$$
p_{2}=p_{4}=0
$$

i.e. to the condition $T \in \mathcal{T}_{1} \oplus \mathcal{T}_{3}$. The same paper shows that on every Riemannian almost product manifold $(M, P, g)$ there exists an unique canonical connection $\nabla^{\prime}$, and it is determined by

$$
g\left(\nabla_{x}^{\prime} y, z\right)=g\left(\nabla_{x} y, z\right)+\frac{1}{4}\{\Phi(x, y, z)-2 \Phi(z, x, y)-\Phi(x, P y, P z)\}
$$

For the torsion $T$ of this connection it is valid

$$
\begin{aligned}
T(x, y, z)=\frac{1}{4}\{\Phi(y, z, x) & -\Phi(z, x, y) \\
& +\Phi(y, P z, P x)+\Phi(P z, x, P y)\} .
\end{aligned}
$$

Proposition 4.2. Let $T$ be the torsion of the canonical connection $\nabla^{\prime}$ on a Riemannian almost product $\mathcal{W}_{3}$-manifold $(M, P, g)$. Then $T$ has the properties

$$
\begin{gathered}
T(P x, y)=-P T(x, y) \\
T(P x, y, z)=T(x, P y, z)=-T(x, y, P z),
\end{gathered}
$$




$$
\begin{gathered}
T(T(P x, P y), z, w)=T(T(P x, y), z, P w) \\
=T(T(x, P y), z, P w)=T(T(x, y), z, w) \\
T=p_{3}, \text { i.e. } T \in \mathcal{T}_{3}
\end{gathered}
$$

and $\nabla^{\prime}$ is determined by

$$
\nabla_{x}^{\prime} y=\nabla_{x} y+\frac{1}{4}\left\{-\left(\nabla_{y} P\right) P x+\left(\nabla_{P y} P\right) x-2\left(\nabla_{x} P\right) P y\right\} .
$$

Proof. By virtue of (4.4), (3.8), (2.3) and (2.4) we obtain (4.5). Then, (4.5), (3.1) and (2.1) imply (4.6) and (4.7). From Proposition 3.3 and condition (4.2) we obtain immediately (4.8). Equalities (3.8) and (4.3) imply (4.9).

Let $\nabla^{\prime}$ be the canonical connection on a Riemannian almost product $\mathcal{W}_{3^{-}}$ manifold $(M, P, g)$. According to (4.9), for the tensor $Q$ and the torsion $T$ of $\nabla^{\prime}$ we have

$$
\begin{gathered}
Q(x, y)=\frac{1}{4}\left\{-\left(\nabla_{y} P\right) P x+\left(\nabla_{P y} P\right) x-2\left(\nabla_{x} P\right) P y\right\}, \\
T(x, y)=-\frac{1}{2}\left\{\left(\nabla_{x} P\right) P y+\left(\nabla_{P x} P\right) y\right\} .
\end{gathered}
$$

Hence, having in mind (4.11), (2.2) and (3.1), we obtain

$$
T(x, y, z)=-\frac{1}{2}\{F(x, P y, z)+F(P x, y, z)\} .
$$

Substituting $y \leftrightarrow z$ into the above, according to (2.3), we get

$$
T(x, z, y)=\frac{1}{2}\{F(x, P y, z)-F(P x, y, z)\} .
$$

Subtracting (4.13) from (4.12) and replacing $y$ with $P y$ in the result, we have

$$
F(x, y, z)=T(x, z, P y)-T(x, P y, z) .
$$

Equalities (4.10), (3.1) and (2.2) imply

$$
Q(x, y, z)=-\frac{1}{4}\{F(y, P x, z)-F(P y, x, z)+2 F(x, P y, z)\} .
$$

Hence, because of (2.3) and (2.4), we conclude that

$$
Q(x, y, z)=-Q(y, x, z)-F(P z, x, y) .
$$

Theorem 4.3. Let $\tau^{\prime}$ and $\tau$ be the scalar curvatures for the canonical connection $\nabla^{\prime}$ and the Levi-Civita connection $\nabla$, respectively, on a Riemannian almost product $\mathcal{W}_{3}$-manifold $(M, P, g)$. Then

$$
\|\nabla P\|^{2}=8\left(\tau^{\prime}-\tau\right)
$$


Proof. According to (2.1) and (2.3), for a Riemannian almost product manifold we have $g^{i j} F\left(P z, e_{i}, e_{j}\right)=0$. Then, from (4.16), after contraction by $x=e_{i}, y=e_{j}$, we obtain

$$
g^{i j} Q\left(e_{i}, e_{j}, z\right)=0 .
$$

Because of $\nabla g^{i j}=0$ and (4.18), we get

$$
g^{i j}\left(\nabla_{x} Q\right)\left(e_{i}, e_{j}, z\right)=0 .
$$

It is known that for the curvature tensors $R^{\prime}$ and $R$ of $\nabla^{\prime}$ and $\nabla$, respectively, the following is valid:

$$
\begin{aligned}
R^{\prime}(x, y, z, w)=R(x, y, z, w) & +\left(\nabla_{x} Q\right)(y, z, w)-\left(\nabla_{y} Q\right)(x, z, w) \\
& +Q(x, Q(y, z), w)-Q(y, Q(x, z), w) .
\end{aligned}
$$

Then from (3.4) and (3.1) it follows that

$$
\begin{aligned}
R^{\prime}(x, y, z, w)= & R(x, y, z, w)+\left(\nabla_{x} Q\right)(y, z, w)-\left(\nabla_{y} Q\right)(x, z, w) \\
& -g(Q(x, w), Q(y, z))+g(Q(y, w), Q(x, z))
\end{aligned}
$$

for a Riemannian almost product manifold $(M, P, g)$. Using a contraction by $x=e_{i}, w=e_{j}$ in (4.20) and combining (3.4), (4.18) and (4.19), we find that the Ricci tensors $\rho^{\prime}$ and $\rho$ for $\nabla^{\prime}$ and $\nabla$ satisfy

$$
\rho^{\prime}(y, z)=\rho(y, z)+g^{i j}\left(\nabla_{e_{i}} Q\right)\left(y, z, e_{j}\right)+g^{i j} g\left(Q\left(y, e_{j}\right), Q\left(e_{i}, z\right)\right) .
$$

Similarly, after a contraction by $y=e_{k}, z=e_{s}$ in (4.21) and according to (4.19), we obtain

$$
\tau^{\prime}=\tau+g^{i j} g^{k s} g\left(Q\left(e_{k}, e_{j}\right), Q\left(e_{i}, e_{s}\right)\right)
$$

for the scalar curvatures $\tau^{\prime}$ and $\tau$ for $\nabla^{\prime}$ and $\nabla$.

The equalities (4.22) and (4.10) imply on a Riemannian $\mathcal{W}_{3}$-manifold $(M, P, g)$ the following equality

$$
g^{i j} g^{k s} g\left(Q\left(e_{k}, e_{j}\right), Q\left(e_{i}, e_{s}\right)\right)=\frac{1}{16} g^{i j} g^{k s} g\left(A_{j k}, A_{s i}\right)
$$

where

$$
A_{j k}=-\left(\nabla_{e_{j}} P\right) P e_{k}+\left(\nabla_{P e_{j}} P\right) e_{k}-2\left(\nabla_{e_{k}} P\right) P e_{j} .
$$

From (4.23), (2.1) and (2.5) we get

$$
g^{i j} g^{k s} g\left(Q\left(e_{k}, e_{j}\right), Q\left(e_{i}, e_{s}\right)\right)=\frac{1}{8}\|\nabla P\|^{2} .
$$

The last equality and (4.22) imply (4.17).

Corollary 4.4. A Riemannian almost product $\mathcal{W}_{3}$-manifold is a Riemannian $P$-manifold if and only if the scalar curvatures for the canonical connection and the Levi-Civita connection are equal. 


\section{Canonical connection on a Riemannian almost product $\mathcal{W}_{3}$-manifold whose curvature tensor is a Riemannian $P$-tensor}

It is known ([9]) that for every linear connection $\nabla^{\prime}$ on a Riemannian manifold $(M, g)$ with a torsion $T$ and a curvature tensor $R^{\prime}$ the following equality (the first Bianchi identity) is valid

$$
\underset{x, y, z}{\mathfrak{S}} R^{\prime}(x, y) z=\underset{x, y, z}{\mathfrak{S}}\left\{\left(\nabla_{x}^{\prime} T\right)(y, z)+T(T(x, y), z)\right\}
$$

Let $\nabla^{\prime}$ is a natural connection on a Riemannian almost product manifold $(M, P, g)$. Then the latter equality and $\nabla^{\prime} g=0$ imply

$$
\underset{x, y, z}{\mathfrak{S}} R^{\prime}(x, y, z, w)=\underset{x, y, z}{\mathfrak{S}}\left\{\left(\nabla_{x}^{\prime} T\right)(y, z, w)+T(T(x, y), z, w)\right\} .
$$

For the curvature tensor $R^{\prime}$ the condition $(2.6)$ is valid. Then $R^{\prime}$ is a Riemannian $P$-tensor if the conditions (2.7) and (2.8) are satisfy for $R^{\prime}$, too. Since $\nabla^{\prime} P=0$ for the natural connection $\nabla^{\prime}$ then (2.8) is valid. The condition (2.7) for $R^{\prime}$ is satisfied according to (5.1) if and only if the following equality is valid

$$
\underset{x, y, z}{\mathfrak{S}}\left\{\left(\nabla_{x}^{\prime} T\right)(y, z, w)+T(T(x, y), z, w)\right\}=0 .
$$

Now, let us consider the case when $(M, P, g)$ belongs to the class $\mathcal{W}_{3}$.

Proposition 5.1. If the curvature tensor of the canonical connection $\nabla^{\prime}$ on a Riemannian almost product $\mathcal{W}_{3}$-manifold $(M, P, g)$ is a Riemannian $P$ tensor, then the following identity for the torsion $T$ of $\nabla^{\prime}$ is valid

$$
T(T(x, y), z, w)=0 .
$$

Proof. We substitute $P z$ for $z$ and $P w$ for $w$ in (5.2). Hence, according to (4.6), we obtain

$$
\begin{aligned}
& \left(\nabla_{x}^{\prime} T\right)(y, z, w)-\left(\nabla_{y}^{\prime} T\right)(z, x, w)+\left(\nabla_{P z}^{\prime} T\right)(x, y, P w) \\
& +T(T(x, y), z, w)+T(T(y, P z), x, w)+T(T(P z, x), y, P w)=0 .
\end{aligned}
$$

We add the last equality to (5.2), and substitute $P x$ for $x$ and $P w$ for $w$ in the result. Then, using (4.6), we get

$$
\begin{aligned}
& \left(\nabla_{z}^{\prime} T\right)(x, y, z)-\left(\nabla_{P z}^{\prime} T\right)(x, P y, w) \\
& +2 T(T(y, z), x, w)+2 T(T(z, x), y, w)=0 .
\end{aligned}
$$

In the latter equality we substitute $P y, P z$ for $y, z$, respectively, and we apply Proposition 4.2. We add the obtained equality to (5.4) and this leads to (5.3). 
Let the curvature tensor of the canonical connection on a Riemannian almost product $\mathcal{W}_{3}$-manifold $(M, P, g)$ is a Riemannian $P$-tensor. Then (5.3) is valid. According to (5.3), (4.14) and the properties of $T$ from Proposition 4.2, we get

$$
F(P y, w, T(z, x))=-T(y, w, T(z, x)) .
$$

Then, using (2.2) and (3.1) we obtain

$$
g\left(T(x, z), T(y, w)+\left(\nabla_{P y} P\right) w\right)=0 .
$$

Since, according to (4.11), we have

$$
T(y, w)=-\frac{1}{2}\left\{\left(\nabla_{y} P\right) P w+\left(\nabla_{P y} P\right) w\right\}
$$

and therefore the following equality is valid

$$
T(y, w)+\left(\nabla_{P y} P\right) w=-\frac{1}{2}\left\{\left(\nabla_{y} P\right) P w-\left(\nabla_{P y} P\right) w\right\} .
$$

By virtue of the latter two equalities and (5.5), we arrive at the following

Theorem 5.2. If the curvature tensor of the canonical connection $\nabla^{\prime}$ on a Riemannian almost product $\mathcal{W}_{3}$-manifold $(M, P, g)$ is a Riemannian P-tensor, then the following identity for the torsion $T$ of $\nabla^{\prime}$ is valid

$$
g\left(\left(\nabla_{x} P\right) P z+\left(\nabla_{P x} P\right) z,\left(\nabla_{P y} P\right) w-\left(\nabla_{y} P\right) P w\right)=0 .
$$

\section{Canonical connection with parallel torsion on a Riemannian almost product $\mathcal{W}_{3}$-manifold}

In this section we consider a canonical connection $\nabla^{\prime}$ with parallel torsion $T$ with respect to $\nabla^{\prime}$ (i.e. $\nabla^{\prime} T=0$ ) on a Riemannian almost product $\mathcal{W}_{3^{-}}$ manifold $(M, P, g)$.

According to the Hayden theorem ([10]) for any natural connection we have

$$
Q(x, y, z)=\frac{1}{2}\{T(x, y, z)-T(y, z, x)+T(z, x, y)\} .
$$

Combining this with (3.2), 3.3), (4.15), leads to the following

Proposition 6.1. Let $\nabla^{\prime}$ be a natural connection on a Riemannian almost product $\mathcal{W}_{3}$-manifold $(M, P, g)$. Then the tensors $T, Q$ and $F$ are parallel or non-parallel at the same time with respect to $\nabla^{\prime}$.

Let $\nabla^{\prime}$ be a natural connection with parallel torsion $T$ on a Riemannian almost product manifold $(M, P, g)$. According to (6.1) we have $\nabla^{\prime} Q=0$. Then, having in mind the formula for the covariant derivative of $Q$, we obtain

$$
x Q(y, z, w)-Q\left(\nabla_{x}^{\prime} y, z, w\right)-Q\left(y, \nabla_{x}^{\prime} z, w\right)-Q\left(y, z, \nabla_{x}^{\prime} w\right)=0 .
$$

Applying the formula for the covariant derivative of $Q$ with respect to $\nabla$ and equalities (3.1), (3.2) and (4.20), we obtain the following 
Lemma 6.2. Let the canonical connection $\nabla^{\prime}$ on a Riemannian almost product manifold $(M, P, g)$ have a parallel torsion $T$. Then for the curvature tensor $R^{\prime}$ of $\nabla^{\prime}$ is valid

$$
\begin{aligned}
R^{\prime}(x, y, z, w)= & R(x, y, z, w)+Q(T(x, y), z, w) \\
& +g(Q(y, z), Q(x, w))-g(Q(x, z), Q(y, w))
\end{aligned}
$$

Let $(M, P, g)$ be a Riemannian almost product $\mathcal{W}_{3}$-manifold whose canonical connection $\nabla^{\prime}$ has a parallel torsion $T$. Then, according to (3.4), (4.16) and (2.2), we have

$$
Q(T(x, y), z, w)=g(Q(z, w), T(x, y))-g\left(\left(\nabla_{P w} P\right) z, T(x, y)\right) .
$$

The last equality and Lemma 6.2 imply the following statement.

Proposition 6.3. Let the canonical connection $\nabla^{\prime}$ on a Riemannian almost product $\mathcal{W}_{3}$-manifold $(M, P, g)$ have a parallel torsion $T$. Then for the curvature tensor $R^{\prime}$ of $\nabla^{\prime}$ is valid

$$
\begin{aligned}
R^{\prime}(x, y, z, w)= & R(x, y, z, w) \\
& +g(Q(y, z), Q(x, w))-g(Q(x, z), Q(y, w)) \\
& +g(Q(z, w), T(x, y))-g\left(\left(\nabla_{P w} P\right) z, T(x, y)\right) .
\end{aligned}
$$

Theorem 6.4. Let the canonical connection $\nabla^{\prime}$ on a Riemannian almost product $\mathcal{W}_{3}$-manifold $(M, P, g)$ have a parallel torsion $T$. Then the curvature tensor $R^{\prime}$ of $\nabla^{\prime}$ is a Riemannian P-tensor.

Proof. Let the canonical connection $\nabla^{\prime}$ on a Riemannian almost product $\mathcal{W}_{3^{-}}$ manifold $(M, P, g)$ have a parallel torsion $T$, i.e. $\nabla^{\prime} T=0$. Then from (5.1) we have

$$
\begin{aligned}
& R^{\prime}(x, y, z, w)+R^{\prime}(y, z, x, w)+R^{\prime}(z, x, y, w) \\
& =T(T(x, y), z, w)+T(T(y, z), x, w)+T(T(z, x), y, w) .
\end{aligned}
$$

We substitute $z \rightarrow P z$ and $w \rightarrow P w$ in (6.2). After that, using property (2.8) for $R^{\prime}$ and the properties of $T$ from Proposition 4.2, we obtain

$$
\begin{aligned}
& R^{\prime}(x, y, z, w)+R^{\prime}(y, P z, P x, w)+R^{\prime}(P z, x, P y, w) \\
& =-T(T(x, y), z, w)+T(T(y, z), x, w)+T(T(z, x), y, w) .
\end{aligned}
$$

We subtract the latter equality from (6.2) and get

$$
\begin{aligned}
& R^{\prime}(z, x, y, w)+R^{\prime}(y, z, x, w)-R^{\prime}(P z, x, P y, w)-R^{\prime}(y, P z, P x, w) \\
& =2 T(T(x, y), z, w) .
\end{aligned}
$$

Bearing in mind the properties of $R^{\prime}$ and $T$, from (6.3) we obtain

$$
\begin{aligned}
& R^{\prime}(z, x, y, w)-R^{\prime}(z, P x, P y, w)-R^{\prime}(P z, x, P y, w)+R^{\prime}(P z, P x, y, w)=0 \\
& R^{\prime}(z, x, y, w)-R^{\prime}(z, P x, P y, w)-R^{\prime}(P z, P x, y, w)+R^{\prime}(P z, x, P y, w)=0
\end{aligned}
$$


which give

$$
R^{\prime}(z, x, y, w)=R^{\prime}(z, P x, P y, w) .
$$

Equality 6.4 implies

$$
R^{\prime}(z, x, y, w)=R^{\prime}(P z, P x, y, w)=R^{\prime}(P z, x, P y, w) .
$$

Applying (6.4) and (6.5) in (6.3), we obtain (5.3). Then from (6.2) it follows condition (2.7) for $R^{\prime}$, i.e. $R^{\prime}$ is a Riemannian $P$-tensor.

\section{Example}

\subsection{A Lie group $G$ as a Riemannian almost product $\mathcal{W}_{3}$-manifold $(G, P, g)$}

Let $G$ be a 4 -dimensional real connected Lie group and $\mathfrak{g}$ be its Lie algebra with a basis $\left\{X_{1}, X_{2}, X_{3}, X_{4}\right\}$.

We introduce a structure $P$ and left invariant metric $g$ as follows

$$
\begin{gathered}
P X_{1}=X_{3}, \quad P X_{2}=X_{4}, \quad P X_{3}=X_{1}, \quad P X_{4}=X_{2}, \\
g\left(X_{i}, X_{j}\right)= \begin{cases}1, & i=j ; \\
0, & i \neq j .\end{cases}
\end{gathered}
$$

Obviously, $P^{2} X_{i}=X_{i}, g\left(P X_{i}, P X_{j}\right)=g\left(X_{i}, X_{j}\right)$ and then $(G, P, g)$ is a Riemannian almost product manifold with $\operatorname{tr} P=0$.

Theorem 7.1. If $(G, P, g)$ has a Killing associated metric $\tilde{g}$, i.e.

$$
g\left(\left[X_{i}, X_{j}\right], P X_{k}\right)+g\left(\left[X_{i}, X_{k}\right], P X_{j}\right)=0,
$$

then $(G, P, g)$ is a Riemannian almost product $\mathcal{W}_{3}$-manifold.

Proof. The well-known formula

$$
\begin{aligned}
2 g\left(\nabla_{X_{i}} X_{j}, X_{k}\right) & =X_{i} g\left(X_{j}, X_{k}\right)+X_{j} g\left(X_{i}, X_{k}\right)-X_{k} g\left(X_{i}, X_{j}\right) \\
& +g\left(\left[X_{i}, X_{j}\right], X_{k}\right)+g\left(\left[X_{k}, X_{i}\right], X_{j}\right)+g\left(\left[X_{k}, X_{j}\right], X_{i}\right)
\end{aligned}
$$

and (7.2) imply

$$
\begin{aligned}
2 g\left(\nabla_{X_{i}} X_{j}, X_{k}\right)=g\left(\left[X_{i}, X_{j}\right], X_{k}\right) & +g\left(\left[X_{k}, X_{i}\right], X_{j}\right) \\
& +g\left(\left[X_{k}, X_{j}\right], X_{i}\right) .
\end{aligned}
$$

Since (7.3) the following equalities are valid

$$
\begin{aligned}
& g\left(\left[X_{k}, X_{i}\right], X_{j}\right)=g\left(P\left[X_{i}, P X_{j}\right], X_{k}\right), \\
& g\left(\left[X_{k}, X_{j}\right], X_{i}\right)=-g\left(P\left[P X_{i}, X_{j}\right], X_{k}\right) .
\end{aligned}
$$

Then from (17.4) we get

$$
\nabla_{X_{i}} X_{j}=\frac{1}{2}\left\{\left[X_{i}, X_{j}\right]+P\left[X_{i}, P X_{j}\right]-P\left[P X_{i}, X_{j}\right]\right\},
$$


which implies

$$
\left(\nabla_{X_{i}} P\right) X_{j}=\frac{1}{2}\left\{\left[P X_{i}, X_{j}\right]-P\left[P X_{i}, P X_{j}\right]\right\} .
$$

Hence, according to (2.2), we obtain

$$
F\left(X_{i}, X_{j}, X_{k}\right)=\frac{1}{2}\left\{g\left(\left[P X_{i}, X_{j}\right], X_{k}\right)+g\left(\left[P X_{i}, X_{k}\right], X_{j}\right)\right\} .
$$

Equality (7.7) implies $\underset{i, j, k}{\mathfrak{S}} F\left(X_{i}, X_{j}, X_{k}\right)=0$. Therefore, $(G, P, g)$ is a Riemannian almost product $\mathcal{W}_{3}$-manifold.

Let $(G, P, g)$ have a Killing associated metric $\tilde{g}$. Then, according to Theorem 7.1 the manifold $(G, P, g)$ is a Riemannian almost product $\mathcal{W}_{3}$-manifold. Applying condition (17.3) and the Jacobi identity for the commutators $\left[X_{i}, X_{j}\right]$, we obtain

$$
\begin{array}{lll}
{\left[X_{1}, X_{2}\right]=\lambda_{1} X_{1}+\lambda_{2} X_{2},} & {\left[X_{1}, X_{3}\right]=\lambda_{3} X_{2}-\lambda_{1} X_{4},} \\
{\left[X_{1}, X_{4}\right]=-\lambda_{3} X_{1}-\lambda_{2} X_{4},} & {\left[X_{2}, X_{3}\right]=\lambda_{4} X_{2}+\lambda_{1} X_{3},} \\
{\left[X_{2}, X_{4}\right]=-\lambda_{4} X_{1}+\lambda_{2} X_{3},} & {\left[X_{3}, X_{4}\right]=\lambda_{3} X_{3}+\lambda_{4} X_{4},}
\end{array}
$$

where $\lambda_{i} \in \mathbb{R}(i=1,2,3,4)$.

Vice verse, if equalities (7.8) are valid for a Riemannian almost product manifold $(G, P, g)$, then we verify directly that the Jacobi identity for the commutators $\left[X_{i}, X_{j}\right]$ is satisfied and the associated metric $\tilde{g}$ is Killing.

Hence, it is valid the following

Theorem 7.2. The manifold $(G, P, g)$ is a Riemannian almost product $\mathcal{W}_{3}$ manifold with a Killing associated metric $\tilde{g}$ if and only if the Lie algebra $\mathfrak{g}$ is determined by conditions (7.8).

Further, $(G, P, g)$ will stand for the Riemannian almost product $\mathcal{W}_{3}$-manifold determined by conditions (7.8).

\subsection{Some geometrical characteristics of the manifold $(G, P, g)$}

According to (7.7), (7.1), (7.2) and (7.8), we get the non-zero components $F_{i j k}=F\left(X_{i}, X_{j}, X_{k}\right)$ of the tensor $F$ :

$$
\begin{aligned}
& 2 F_{114}=-2 F_{123}=2 F_{312}=-2 F_{334}=-F_{411}=F_{433}=\lambda_{1}, \\
& -2 F_{223}=2 F_{241}=F_{322}=-F_{344}=-2 F_{412}=2 F_{434}=\lambda_{2}, \\
& -2 F_{112}=2 F_{134}=F_{211}=-F_{233}=-2 F_{314}=2 F_{332}=\lambda_{3}, \\
& -F_{122}=F_{144}=2 F_{221}=-2 F_{234}=-2 F_{414}=2 F_{432}=\lambda_{4} .
\end{aligned}
$$

The rest of the non-zero components are obtained by the property $F_{i j k}=$ $F_{i k j}$. 
By virtue of (7.6) and (7.8) we compute the components of $\nabla P$ :

$$
\begin{aligned}
& 2\left(\nabla_{X_{1}} P\right) X_{1}=-2\left(\nabla_{X_{3}} P\right) X_{3}=-\lambda_{3} X_{2}+\lambda_{1} X_{4}, \\
& 2\left(\nabla_{X_{2}} P\right) X_{2}=-2\left(\nabla_{X_{4}} P\right) X_{4}=\lambda_{4} X_{1}-\lambda_{2} X_{3}, \\
& 2\left(\nabla_{X_{1}} P\right) X_{3}=-2\left(\nabla_{X_{3}} P\right) X_{1}=-\lambda_{1} X_{2}+\lambda_{3} X_{4}, \\
& 2\left(\nabla_{X_{2}} P\right) X_{4}=-2\left(\nabla_{X_{4}} P\right) X_{2}=\lambda_{2} X_{1}-\lambda_{4} X_{3}, \\
& 2\left(\nabla_{X_{1}} P\right) X_{2}=-\lambda_{3} X_{1}-2 \lambda_{4} X_{2}-\lambda_{1} X_{3}, \\
& 2\left(\nabla_{X_{1}} P\right) X_{4}=\lambda_{1} X_{1}+\lambda_{3} X_{3}+2 \lambda_{4} X_{4}, \\
& 2\left(\nabla_{X_{2}} P\right) X_{1}=2 \lambda_{3} X_{1}+\lambda_{4} X_{2}+\lambda_{2} X_{4}, \\
& 2\left(\nabla_{X_{2}} P\right) X_{3}=-\lambda_{2} X_{2}-2 \lambda_{3} X_{3}-\lambda_{4} X_{4}, \\
& 2\left(\nabla_{X_{3}} P\right) X_{2}=\lambda_{1} X_{1}+2 \lambda_{2} X_{2}+\lambda_{3} X_{3}, \\
& 2\left(\nabla_{X_{3}} P\right) X_{4}=-\lambda_{3} X_{1}-\lambda_{1} X_{3}-2 \lambda_{2} X_{4}, \\
& 2\left(\nabla_{X_{4}} P\right) X_{1}=-2 \lambda_{1} X_{1}-\lambda_{2} X_{2}-\lambda_{4} X_{4}, \\
& 2\left(\nabla_{X_{4}} P\right) X_{3}=\lambda_{4} X_{2}+2 \lambda_{1} X_{3}+\lambda_{2} X_{4} .
\end{aligned}
$$

Using these components and (2.5), we obtain the square norm of $\nabla P$ :

$$
\|\nabla P\|^{2}=4\left(\lambda_{1}^{2}+\lambda_{2}^{2}+\lambda_{3}^{2}+\lambda_{4}^{2}\right) .
$$

The components of the Levi-Civita connection $\nabla$ we compute by (7.5) and (7.8):

$$
\begin{aligned}
& \nabla_{X_{1}} X_{1}=-\nabla_{X_{3}} X_{3}=-\lambda_{1} X_{2}+\lambda_{3} X_{4}, \\
& \nabla_{X_{2}} X_{2}=-\nabla_{X_{4}} X_{4}=\lambda_{2} X_{1}-\lambda_{4} X_{3}, \\
& 2 \nabla_{X_{1}} X_{3}=-2 \nabla_{X_{3}} X_{1}=\lambda_{3} X_{2}-\lambda_{1} X_{4}, \\
& 2 \nabla_{X_{2}} X_{4}=-2 \nabla_{X_{4}} X_{2}=-\lambda_{4} X_{1}+\lambda_{2} X_{3}, \\
& 2 \nabla_{X_{1}} X_{2}=2 \lambda_{1} X_{1}-\lambda_{3} X_{3}+\lambda_{4} X_{4}, \\
& 2 \nabla_{X_{1}} X_{4}=-2 \lambda_{3} X_{1}-\lambda_{4} X_{2}+\lambda_{1} X_{3}, \\
& 2 \nabla_{X_{2}} X_{1}=-2 \lambda_{2} X_{2}-\lambda_{3} X_{3}+\lambda_{4} X_{4}, \\
& 2 \nabla_{X_{2}} X_{3}=\lambda_{3} X_{1}+2 \lambda_{4} X_{2}-\lambda_{2} X_{4}, \\
& 2 \nabla_{X_{3}} X_{2}=\lambda_{3} X_{1}-2 \lambda_{1} X_{3}-\lambda_{2} X_{4}, \\
& 2 \nabla_{X_{3}} X_{4}=-\lambda_{1} X_{1}+\lambda_{2} X_{2}+2 \lambda_{3} X_{3}, \\
& 2 \nabla_{X_{4}} X_{1}=-\lambda_{4} X_{2}+\lambda_{1} X_{3}+2 \lambda_{2} X_{4}, \\
& 2 \nabla_{X_{4}} X_{3}=-\lambda_{1} X_{1}+\lambda_{2} X_{2}-2 \lambda_{4} X_{4} .
\end{aligned}
$$

By virtue of (7.12) and (7.8), from the formula

$$
\begin{aligned}
R\left(X_{i}, X_{j}, X_{k}, X_{s}\right)=g\left(\nabla_{X_{i}} \nabla_{X_{j}} X_{k}, X_{s}\right) & -g\left(\nabla_{X_{j}} \nabla_{X_{i}} X_{k}, X_{s}\right) \\
& -g\left(\nabla_{\left[X_{i}, X_{j}\right]} X_{k}, X_{s}\right)
\end{aligned}
$$


we get the following non-zero components $R_{i j k s}=R\left(X_{i}, X_{j}, X_{k}, X_{s}\right)$ of the tensor $R$ :

$$
\begin{aligned}
& R_{1234}=\lambda_{1}^{2}+\lambda_{2}^{2}, \\
& R_{1331}=\frac{1}{4}\left(\lambda_{3}^{2}+\lambda_{1}^{2}\right), \quad R_{2442}=\frac{1}{4}\left(\lambda_{4}^{2}+\lambda_{2}^{2}\right), \\
& R_{1441}=\frac{1}{4}\left(\lambda_{4}^{2}+\lambda_{1}^{2}-4 \lambda_{3}^{2}-4 \lambda_{2}^{2}\right) \\
& R_{2332}=\frac{1}{4}\left(\lambda_{3}^{2}+\lambda_{2}^{2}-4 \lambda_{4}^{2}-4 \lambda_{1}^{2}\right) \\
& R_{3443}=\frac{1}{4}\left(\lambda_{1}^{2}+\lambda_{2}^{2}-4 \lambda_{3}^{2}-4 \lambda_{4}^{2}\right) \\
& R_{1221}=\frac{1}{4}\left(\lambda_{3}^{2}+\lambda_{4}^{2}-4 \lambda_{1}^{2}-4 \lambda_{2}^{2}\right) \\
& R_{1241}=R_{3243}=\frac{1}{4}\left(4 \lambda_{2} \lambda_{4}+3 \lambda_{1} \lambda_{3}\right) \\
& R_{2132}=R_{4134}=\frac{1}{4}\left(4 \lambda_{1} \lambda_{3}+3 \lambda_{2} \lambda_{4}\right) \\
& R_{1231}=R_{4234}=\frac{1}{4}\left(3 \lambda_{1} \lambda_{4}-2 \lambda_{2} \lambda_{3}\right) \\
& R_{2142}=R_{3143}=\frac{1}{4}\left(3 \lambda_{2} \lambda_{3}-2 \lambda_{1} \lambda_{4}\right) \\
& R_{1341}=R_{2342}=\frac{1}{4}\left(3 \lambda_{3} \lambda_{4}-2 \lambda_{1} \lambda_{2}\right), \\
& R_{3123}=R_{4124}=\frac{1}{4}\left(3 \lambda_{1} \lambda_{2}-2 \lambda_{3} \lambda_{4}\right) .
\end{aligned}
$$

The rest of the non-zero components are obtained by the properties

$$
R_{i j k s}=R_{k s i j}, \quad R_{i j k s}=-R_{j i k s}=-R_{i j s k} .
$$

Using (7.13) for the non-zero components $\rho_{i j}=\rho\left(X_{i}, X_{j}\right)$ of the Ricci tensor $\rho$ we compute:

$$
\begin{array}{lll}
\rho_{11}=\frac{1}{2}\left(-\lambda_{3}^{2}-\lambda_{1}^{2}+\lambda_{4}^{2}-4 \lambda_{2}^{2}\right), & \rho_{34}=\frac{1}{2}\left(3 \lambda_{3} \lambda_{4}-2 \lambda_{1} \lambda_{2}\right), \\
\rho_{22}=\frac{1}{2}\left(-\lambda_{4}^{2}-\lambda_{2}^{2}+\lambda_{3}^{2}-4 \lambda_{1}^{2}\right), & \rho_{14}=\frac{1}{2}\left(3 \lambda_{2} \lambda_{3}-2 \lambda_{1} \lambda_{4}\right), \\
\rho_{33}=\frac{1}{2}\left(-\lambda_{1}^{2}+\lambda_{2}^{2}-\lambda_{3}^{2}-4 \lambda_{4}^{2}\right), & \rho_{23}=\frac{1}{2}\left(3 \lambda_{1} \lambda_{4}-2 \lambda_{2} \lambda_{3}\right), \\
\rho_{44}=\frac{1}{2}\left(-\lambda_{2}^{2}+\lambda_{1}^{2}-\lambda_{4}^{2}-4 \lambda_{3}^{2}\right), & \rho_{24}=\frac{1}{2}\left(4 \lambda_{2} \lambda_{4}+3 \lambda_{1} \lambda_{3}\right), \\
\rho_{12}=\frac{1}{2}\left(-3 \lambda_{1} \lambda_{2}+2 \lambda_{3} \lambda_{4}\right), & \rho_{13}=\frac{1}{2}\left(4 \lambda_{1} \lambda_{3}+3 \lambda_{2} \lambda_{4}\right) .
\end{array}
$$

The rest of the non-zero components are obtained by the property $\rho_{i j}=\rho_{j i}$. By (7.14) we obtain the scalar curvature $\tau$ for the connection $\nabla$ :

$$
\tau=-\frac{5}{2}\left(\lambda_{1}^{2}+\lambda_{2}^{2}+\lambda_{3}^{2}+\lambda_{4}^{2}\right)
$$

For the Riemannian sectional curvatures of the $P$-invariant basis 2-planes $\left(X_{1}, X_{3}\right)$ and $\left(X_{2}, X_{4}\right)$, i.e. for the invariant sectional curvatures of the basis 2-planes, we get

$$
k_{13}=\frac{1}{4}\left(\lambda_{1}^{2}+\lambda_{3}^{2}\right), \quad k_{24}=\frac{1}{4}\left(\lambda_{2}^{2}+\lambda_{4}^{2}\right) .
$$


The sectional curvatures of the rest of the basis 2-planes, i.e. the anti-invariant sectional curvatures of the basis 2-planes, are:

$$
\begin{aligned}
& k_{12}=\frac{1}{4}\left(\lambda_{3}^{2}+\lambda_{4}^{2}-4 \lambda_{1}^{2}-4 \lambda_{2}^{2}\right), \\
& k_{14}=\frac{1}{4}\left(\lambda_{1}^{2}+\lambda_{4}^{2}-4 \lambda_{3}^{2}-4 \lambda_{2}^{2}\right), \\
& k_{23}=\frac{1}{4}\left(\lambda_{2}^{2}+\lambda_{3}^{2}-4 \lambda_{4}^{2}-4 \lambda_{1}^{2}\right), \\
& k_{34}=\frac{1}{4}\left(\lambda_{1}^{2}+\lambda_{2}^{2}-4 \lambda_{3}^{2}-4 \lambda_{4}^{2}\right) .
\end{aligned}
$$

Conditions (7.16) and (7.17) imply the following

Proposition 7.3. The manifold $(G, P, g)$ has:

(i) a constant invariant sectional curvature if and only if $\lambda_{1}^{2}-\lambda_{2}^{2}+\lambda_{3}^{2}-\lambda_{4}^{2}=$ 0 ;

(ii) a constant anti-invariant sectional curvature if and only if $\lambda_{1}^{2}=\lambda_{3}^{2}$, $\lambda_{2}^{2}=\lambda_{4}^{2}$

(iii) a constant sectional curvature if and only if $\lambda_{1}^{2}=\lambda_{2}^{2}=\lambda_{3}^{2}=\lambda_{4}^{2}$.

According to (7.8), (7.9) and (7.15), it is valid the following

Proposition 7.4. The following propositions are equivalent:

(i) $\lambda_{1}=\lambda_{2}=\lambda_{3}=\lambda_{4}=0$;

(ii) the Lie algebra $\mathfrak{g}$ is Abelian, i.e. $\left[X_{i}, X_{j}\right]=0(i, j=1,2,3,4)$;

(iii) $(G, P, g)$ is a Riemannian $P$-manifold, i.e. $F=0$;

(iv) $(G, P, g)$ is a scalar flat manifold with respect to $\nabla$, i.e. $\tau=0$.

\subsection{The canonical connection on $(G, P, g)$}

Further in our considerations we exclude the trivial case of Proposition 7.4 for the Riemannian almost product $\mathcal{W}_{3}$-manifold $(G, P, g)$.

By virtue of (4.9), (17.9) and (7.12) for the components of the canonical connection $\nabla^{\prime}$ on $(G, P, g)$ we obtain:

$$
\begin{aligned}
& 2 \nabla_{X_{1}}^{\prime} X_{1}=-2 \nabla_{X_{3}}^{\prime} X_{3}=-\lambda_{1} X_{2}+\lambda_{3} X_{4}, \\
& 2 \nabla_{X_{2}}^{\prime} X_{2}=-2 \nabla_{X_{4}}^{\prime} X_{4}=\lambda_{2} X_{1}-\lambda_{4} X_{3}, \\
& 2 \nabla_{X_{1}}^{\prime} X_{2}=-2 \nabla_{X_{3}}^{\prime} X_{4}=\lambda_{1} X_{1}-\lambda_{3} X_{3}, \\
& 2 \nabla_{X_{1}}^{\prime} X_{3}=-2 \nabla_{X_{3}}^{\prime} X_{1}=\lambda_{3} X_{2}-\lambda_{1} X_{4}, \\
& 2 \nabla_{X_{1}}^{\prime} X_{4}=-2 \nabla_{X_{3}}^{\prime} X_{2}=-\lambda_{3} X_{1}+\lambda_{1} X_{3}, \\
& 2 \nabla_{X_{2}}^{\prime} X_{1}=-2 \nabla_{X_{4}}^{\prime} X_{3}=-\lambda_{2} X_{2}+\lambda_{4} X_{4}, \\
& 2 \nabla_{X_{2}}^{\prime} X_{3}=-2 \nabla_{X_{4}}^{\prime} X_{1}=\lambda_{4} X_{2}-\lambda_{2} X_{4}, \\
& 2 \nabla_{X_{2}}^{\prime} X_{4}=-2 \nabla_{X_{4}}^{\prime} X_{2}=-\lambda_{4} X_{1}+\lambda_{2} X_{3} .
\end{aligned}
$$

Proposition 7.5. The curvature tensor of the canonical connection on $(G, P, g)$ is a Riemannian P-tensor if and only if the following conditions are valid:

$$
\lambda_{1}=\varepsilon \lambda_{3}, \quad \lambda_{2}=\varepsilon \lambda_{4}, \quad \varepsilon= \pm 1 .
$$


Proof. Obviously the curvature tensor $R^{\prime}$ of the canonical connection is a Riemannian $P$-tensor if and only if property (2.7) is satisfied for $R^{\prime}$. Condition (2.7) for $R^{\prime}$ is valid if and only if the following conditions are satisfied:

$$
\underset{1,2,3}{\mathfrak{S}} R_{123}^{\prime}=\underset{1,2,4}{\mathfrak{S}} R_{124}^{\prime}=\underset{1,3,4}{\mathfrak{S}} R_{134}^{\prime}=\underset{2,3,4}{\mathfrak{S}} R_{234}^{\prime}=0
$$

where $R_{i j k}^{\prime}$ are the components of $R^{\prime}$.

Using (7.18) and (7.8), we obtain:

$$
\begin{aligned}
& R_{123}^{\prime}=-\frac{1}{2}\left\{\left(\lambda_{1} \lambda_{3}+\lambda_{2} \lambda_{4}\right) X_{2}-\left(\lambda_{1}^{2}+\lambda_{2}^{2}\right) X_{4}\right\}, \\
& R_{231}^{\prime}=-\frac{1}{2}\left\{\left(\lambda_{1} \lambda_{3}+\lambda_{2} \lambda_{4}\right) X_{2}+\left(\lambda_{1}^{2}+\lambda_{4}^{2}\right) X_{4}\right\}, \\
& R_{312}^{\prime}=\frac{1}{2}\left\{\left(\lambda_{2} \lambda_{3}-\lambda_{1} \lambda_{4}\right) X_{1}+\left(\lambda_{1} \lambda_{2}-\lambda_{3} \lambda_{4}\right) X_{3}\right\}, \\
& R_{124}^{\prime}=\frac{1}{2}\left\{\left(\lambda_{1} \lambda_{3}+\lambda_{2} \lambda_{4}\right) X_{1}-\left(\lambda_{1}^{2}+\lambda_{2}^{2}\right) X_{3}\right\}, \\
& R_{241}^{\prime}=\frac{1}{2}\left\{\left(\lambda_{2} \lambda_{3}-\lambda_{1} \lambda_{4}\right) X_{2}-\left(\lambda_{1} \lambda_{2}-\lambda_{3} \lambda_{4}\right) X_{4}\right\}, \\
& R_{412}^{\prime}=-\frac{1}{2}\left\{\left(\lambda_{1} \lambda_{3}+\lambda_{2} \lambda_{4}\right) X_{1}-\left(\lambda_{2}^{2}+\lambda_{3}^{2}\right) X_{3}\right\}, \\
& R_{134}^{\prime}=\frac{1}{2}\left\{\left(\lambda_{3} \lambda_{4}-\lambda_{1} \lambda_{2}\right) X_{1}+\left(\lambda_{1} \lambda_{4}-\lambda_{2} \lambda_{3}\right) X_{3}\right\}, \\
& R_{341}^{\prime}=-\frac{1}{2}\left\{\left(\lambda_{4}^{2}-\lambda_{3}^{2}\right) X_{2}+\left(\lambda_{2} \lambda_{4}+\lambda_{1} \lambda_{3}\right) X_{4}\right\}, \\
& R_{413}^{\prime}=-\frac{1}{2}\left\{\left(\lambda_{2}^{2}+\lambda_{3}^{2}\right) X_{2}-\left(\lambda_{2} \lambda_{4}+\lambda_{1} \lambda_{3}\right) X_{4}\right\}, \\
& R_{234}^{\prime}=-\frac{1}{2}\left\{-\left(\lambda_{1}^{2}+\lambda_{4}^{2}\right) X_{1}+\left(\lambda_{1} \lambda_{3}+\lambda_{2} \lambda_{4}\right) X_{3}\right\}, \\
& R_{342}^{\prime}=-\frac{1}{2}\left\{\left(\lambda_{3}^{2}+\lambda_{4}^{2}\right) X_{1}-\left(\lambda_{1} \lambda_{3}+\lambda_{2} \lambda_{4}\right) X_{3}\right\}, \\
& R_{423}^{\prime}=-\frac{1}{2}\left\{\left(\lambda_{3} \lambda_{4}-\lambda_{1} \lambda_{2}\right) X_{2}+\left(\lambda_{2} \lambda_{3}-\lambda_{1} \lambda_{4}\right) X_{4}\right\} .
\end{aligned}
$$

Hence it follows directly that condition (2.7) is valid for $R^{\prime}$ if and only if (7.19) is satisfied.

Proposition 7.3 and Proposition 7.5 imply the following

Corollary 7.6. If the curvature tensor of the canonical connection on $(G, P, g)$ is a Riemannian P-tensor, then $(G, P, g)$ is a manifold of constant antiinvariant sectional curvature.

Proposition 7.7. The canonical connection on $(G, P, g)$ has a parallel torsion if and only if the curvature tensor of this connection is a Riemannian Ptensor.

Proof. By virtue of (4.11) and (7.10) we obtain the non-zero components $T_{i j}=T\left(X_{i}, X_{j}\right)$ of the torsion $T$ for the canonical connection $\nabla^{\prime}$ on $(G, P, g)$ :

$$
\begin{aligned}
& T_{12}=T_{34}=-\frac{1}{2}\left(\lambda_{1} X_{1}+\lambda_{2} X_{2}+\lambda_{3} X_{3}+\lambda_{4} X_{4}\right), \\
& T_{14}=-T_{23}=\frac{1}{2}\left(\lambda_{3} X_{1}+\lambda_{4} X_{2}+\lambda_{1} X_{3}+\lambda_{2} X_{4}\right) .
\end{aligned}
$$

Obviously, the equality $T_{14}=P T_{12}$ is valid. Then, bearing in mind that $\nabla^{\prime}$ is a natural connection, we have $\left(\nabla_{X_{i}}^{\prime} T\right)\left(X_{1}, X_{4}\right)=-P\left(\nabla_{X_{i}}^{\prime} T\right)\left(X_{1}, X_{2}\right)$. From the latter condition and (7.20) it is clear that the condition $\nabla^{\prime} T_{12}=0$ is sufficient for the obtaining of the condition $\nabla^{\prime} T=0$. 
From (7.20) we compute the following:

$$
\begin{aligned}
& \left(\nabla_{1}^{\prime} T\right)_{12}=-\frac{1}{4}\left\{\left(\lambda_{1} \lambda_{2}-\lambda_{3} \lambda_{4}\right) X_{1}+\left(\lambda_{3}^{2}-\lambda_{1}^{2}\right) X_{2}+\left(\lambda_{1} \lambda_{4}-\lambda_{2} \lambda_{3}\right) X_{3}\right\}, \\
& \left(\nabla_{2}^{\prime} T\right)_{12}=-\frac{1}{4}\left\{\left(\lambda_{2}^{2}-\lambda_{4}^{2}\right) X_{1}+\left(\lambda_{3} \lambda_{4}-\lambda_{1} \lambda_{2}\right) X_{2}+\left(\lambda_{1} \lambda_{4}-\lambda_{2} \lambda_{3}\right) X_{4}\right\}, \\
& \left(\nabla_{3}^{\prime} T\right)_{12}=-\frac{1}{4}\left\{\left(\lambda_{2} \lambda_{3}-\lambda_{1} \lambda_{4}\right) X_{1}+\left(\lambda_{1} \lambda_{2}-\lambda_{3} \lambda_{4}\right) X_{3}+\left(\lambda_{1}^{2}-\lambda_{3}^{2}\right) X_{4}\right\}, \\
& \left(\nabla_{4}^{\prime} T\right)_{12}=-\frac{1}{4}\left\{\left(\lambda_{2} \lambda_{3}-\lambda_{1} \lambda_{4}\right) X_{2}+\left(\lambda_{4}^{2}-\lambda_{2}^{2}\right) X_{3}+\left(\lambda_{1} \lambda_{2}-\lambda_{3} \lambda_{4}\right) X_{4}\right\},
\end{aligned}
$$

where $\left(\nabla_{i}^{\prime} T\right)_{12}=\left(\nabla_{X_{i}}^{\prime} T\right)\left(X_{1}, X_{2}\right)$. Hence $\nabla^{\prime} T_{12}=0$ if and only if (7.19) is valid, i.e. if and only if the curvature tensor of $\nabla^{\prime}$ is a Riemannian $P$ tensor.

From Theorem 4.3, equalities (7.11) and (7.15), we obtain the following

Proposition 7.8. The manifold $(G, P, g)$ has negative scalar curvatures with respect to the Levi-Civita connection $\nabla$ and the canonical connection $\nabla^{\prime}$, namely

$$
\tau=-\frac{5}{2}\left(\lambda_{1}^{2}+\lambda_{2}^{2}+\lambda_{3}^{2}+\lambda_{4}^{2}\right), \quad \tau^{\prime}=-2\left(\lambda_{1}^{2}+\lambda_{2}^{2}+\lambda_{3}^{2}+\lambda_{4}^{2}\right) .
$$

\section{References}

[1] Lichnerowicz, A.: Généralization de la géométrie kählerienne globale. Coll. de Géom. diff. Louvain 16, no. 2, 99-122 (1955)

[2] Lichnerowicz, A.: Un théorème sur les espaces homogènes complexes. Arch. Math. 5, 207-215 (1954)

[3] Gray, A., Barros, M., Naveira, A., Vanheke, L.: The Chern numbers of holomorphic vector bundles and formally holomorphic connections of complex vector bundles over almost complex manifolds. P. reine andew. Math. 314, 84-98 (1980)

[4] Mihova, V.: Cannonical connections and the cannonical conformal group on a Riemannian almost product manifold. Serdica Math. P. 15, 351-358 (1989)

[5] Yano, K.: Differential geometry of complex and almost complex spaces, Pergamon press (1965)

[6] Naveira, A. M.: A classification of Riemannian almost product manifolds. Rend. Math. 3, 577-592 (1983)

[7] Staikova, M., Gribachev, K.: Canonical connections and their conformal invariants on Riemannian P-manifolds. Serdica Math. P. 18, 150-161 (1992)

[8] Mekerov, D.: On Riemannian almost product manifolds with nonintegrable structure. J. Geom. 89, no. 1-2, 119-129 (2008)

[9] Kobayashi, S., Nomizu, K.: Foundations of differential geometry, vol. 1, Intersci. Publ. (1963).

[10] Hayden, H.: Subspaces of a space with torsion. Proc. London Math. Soc. 34, 27-50 (1934) 


\author{
Dobrinka Gribacheva \\ University of Plovdiv \\ Faculty of Mathematics and Informatics \\ 236 Bulgaria blvd \\ Plovdiv 4003 \\ Bulgaria \\ e-mail: dobrinka@uni-plovdiv.bg \\ Dimitar Mekerov \\ University of Plovdiv \\ Faculty of Mathematics and Informatics \\ 236 Bulgaria blvd \\ Plovdiv 4003 \\ Bulgaria \\ e-mail: mircho@uni-plovdiv.bg
}

